\title{
EAR BIOMETRICS: ONE MORE STEP TOWARDS SOCIAL DISTANCING
}

\author{
Mrs.Mansi Talreja \\ Department of Computer \\ Engineering, V.E.S.I.T, \\ Chembur, Maharashtra, India \\ Ashish Sukhani \\ Department of Computer \\ Engineering, V.E.S.I.T, \\ Chembur, Maharashtra, India
}

\author{
Bhavika Motiramani \\ Department of Computer \\ Engineering, V.E.S.I.T, \\ Chembur, Maharashtra, India
}

\author{
Muskan Asrani \\ Department of Computer \\ Engineering, V.E.S.I.T, \\ Chembur, Maharashtra, India \\ Manas Mangaonkar \\ Department of Computer \\ Engineering, V.E.S.I.T, \\ Chembur, Maharashtra, India
}

\begin{abstract}
Biometrics deals with identification of individuals based on their characteristics. In traditional systems people use to remember passwords, PIN's, tokens for identification. But in recent years biometrics have gained a lot of popularity due to advancement in technology and the secure environment. Variety of biometrics have been developed in recent years such as fingerprint, iris, retinal. Ears are considered as the future of biometrics due to its properties of Universality, which means that every person should have it. Uniqueness, which indicates that no two persons should behave the same characteristic. Permanence, which means that this characteristic does not change over time. Collectability, which indicates that the characteristic can be measured quantitatively. Ears are comparatively large in size and hence are more visible and also their shape does not change radically over a period of time and so are their images easy to be captured.
\end{abstract}

Keywords- Ear biometrics, pre processing, feature extraction, edge detection, grayscale.

\section{INTRODUCTION}

Biometrics(bio:life,metric:measure) came into existence from the early 1970's. Traditionally people used to remember PIN's, passwords for identification purposes but it used to get stolen or forgotten so biometric came into existence. Nowadays biometrics is considered as one of the best systems for identification of individuals in terms of security and accuracy. There have been many biometric systems in existence. There are some limitations with these systems. The problem with fingerprint recognition systems is the scanner sometimes does not identify the person and the reason can be the scanner is sensitive to moisture, oil, sweat and also due to some medical conditions some people might have suffered loss of fingers. In the iris recognition system, the scanning cannot be done from a distance as iris is small in size so it needs to be done closely which leads to deformation of iris non elastically which can lead to some medical issues. In the retinal recognition system, the infrared light beam is directly being shone onto eyes which is harmful for eyes. Due to these limitations and to overcome all this we got motivated to develop a biometric system as an ear and also as this system will be a non contacting system which will also be a safe system.

This system can be used in two modes Identification and Verification.

- Identification: To identify an individual among all the users from the database.

- Verification: To verify the identified user.

Ear recognition is done in 3 parts:

1. Preprocessing: To crop and normalize the clicked image and convert it into gray scale by reducing noise.

2. Feature Extraction: In this step the desired features of the ear are extracted.

3. Ear matching: After feature extraction the ear matching is done.

\section{LITERATURE REVIEW}

The following section gives you a literature review of ear biometrics based on different algorithms and techniques used.

Ear based biometric authentication system by Prof Bandyopadhyay ${ }^{[1]}$ :

They have used the method of correlation along with pre preprocessing method in MATLAB. There are several steps in this: Firstly the image is captured and then cropped to take out only the outer shape of the ear. After this the captured image is 
converted into Grayscale image. With the help of a canny edge detector the edge detection is done to remove noise from the image. Segmentation of the image is done followed by histogram equalization to adjust image intensities. Matching is done using correlation to compare two images pixel by pixel and then it gives the result.

\section{Human Ear Recognition using Geometrical Features by Hesham Elmahdy ${ }^{[2]}$ :}

Their approach is as follows:

Preprocessing to resize the images to have the same size of images. After this they detected the ear using a snake model. Edge detection is done to remove noisy edges using a canny edge detector and then converted into binary images. Post processing of the image is done by applying some morphological operations. Feature Extraction is done to get minimum Euclidean distance between pixels and then classification is done. Basically they have used 3 classifiers Naive, nearest neighbour and $\mathrm{K}$ nearest

neighbour out of which $\mathrm{KNN}$ was the most accurate classifier which gives the result.

\section{Human Recognition through ear biometrics using average} ear approach by Gopal Singh Tandel ${ }^{[3]}$

Their system is based on 5 steps :

1. Firstly the image of the ear is captured and to store it to store it in a database for the data set.

2. After this normalization of the images is done for images to be in equal size which can be done by resizing or cropping of the image.

3. Feature Extraction and preprocessing of the image is done to extract the features of ear such as helix, lobe, anti helix etc using Canny edge detector. It is done by first converting the image to gray scale image and passing it to the detector and the ear signatures are obtained and are saved as binary images.

4. Template formation of the ear image is done for comparing the ear signatures.

5. After this the templates are passed to a proposed algorithm which says that count the number of 1's from the template and read the ear signatures to be compared, perform bitwise logical OR operation and on the basis of number of 1's the result is being displayed whether the user is recognized or not.

\section{Ear Recognition Techniques for Biometrics using Digital Image Processing by Dayanand B.Gore ${ }^{[4]}$}

In this paper they have put forward the idea for their system i.e it is the review paper. They have given proposed methodology for the system and their steps are as follows:

1. Capturing of the image is done and the image is captured and considered as the input.

2. Captured images are given for preprocessing by which they can perform image enhancement, filtering.

3. After this Feature extraction will be done by edge detection, segmentation and then classification is done.

4. Moving to future scope they specified the MATLAB tool for image processing and also Prewitt detection method for getting best results when the images are captured properly in accurate light conditions.

\section{SYSTEM ARCHITECTURE}

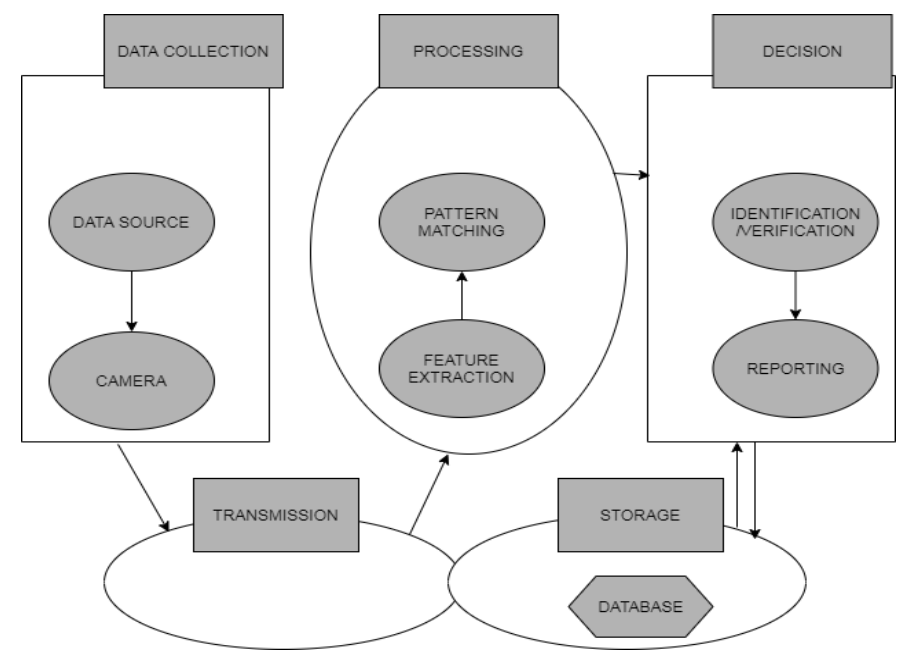

Fig 1: System Architecture

Fig 1 shows the system architecture for our system. It has three main phases and two secondary phases 1) Data Collection 2) Processing 3) Decision 4) Transmission

5) Storage (having database).

In the first phase the image is captured from a digital Camera from the source of data and it is transmitted by transmission phase to processing phase where there are two other parts that is pattern matching and feature extraction. In Feature Extraction the features are extracted or determined for learning by determining the pixels of the image and after then the pattern matching is done by comparing the image parts or pixels of the image and stored in the database and also for authentication purpose the image from preprocessing phase is transmitted to decision phase where the verification/identification of the image is done and the reporting of the result is done.

\section{PROPOSED SOLUTION}

In Fig 2 Proposed Model for our system is shown : It will be an Android Application which will be having two modes i.e

1. Registration Mode

2. Recognition Mode 


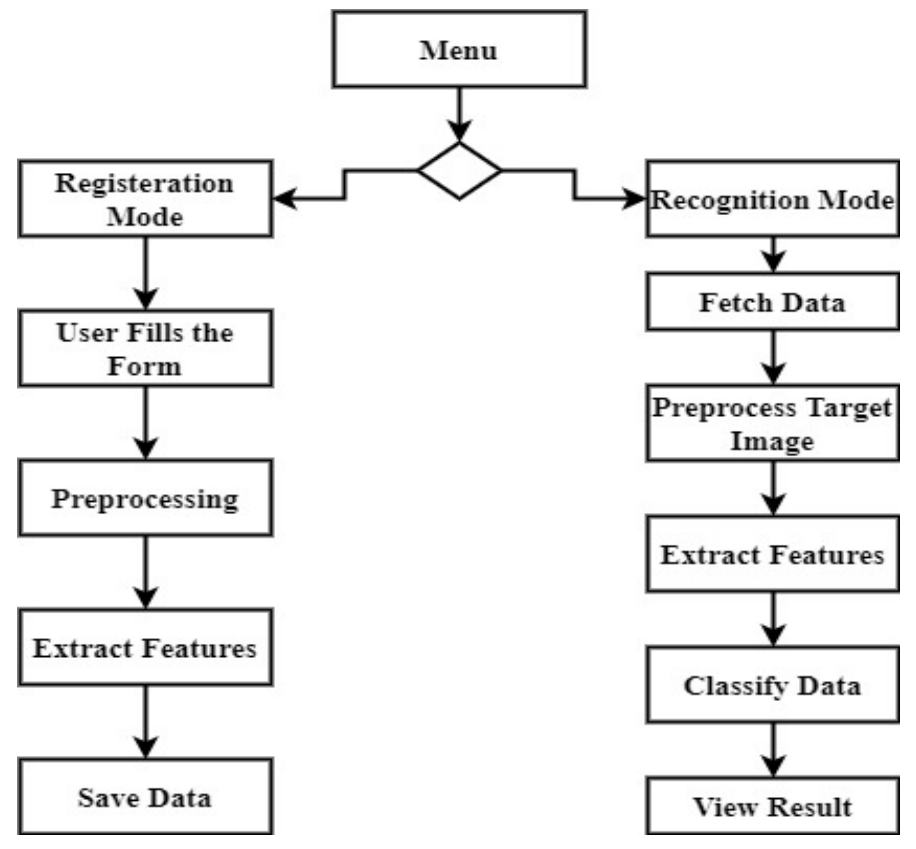

Fig 2:Proposed Model Diagram

a) In Registration mode the user will have to register for the system by filling the registration form with the required information. After the user fills the form, the information from the registration form goes for preprocessing along with the captured image of the ear to be registered in the database. In preprocessing the image goes for resizing, grayscale conversion, edge detection. By preprocessing the image data quality is improved and then it will go for feature extraction where the required features of the ear are extracted for extracting pixels of the image and then this image is stored i.e passed to save data.

b) In Recognition mode the data (i.e the image of the ear) is fetched from the database heaving images. Preprocessing is done for the targeted image: Segmentation, feature extraction, histogram equalization are done. After this the features are extracted from the fetched image and then the classification of the data is done that is the required information is extracted and classified and then the result is being displayed that is whether the image of the ear is recognized or not recognized as an authorized user.

\section{METHODOLOGY USED}

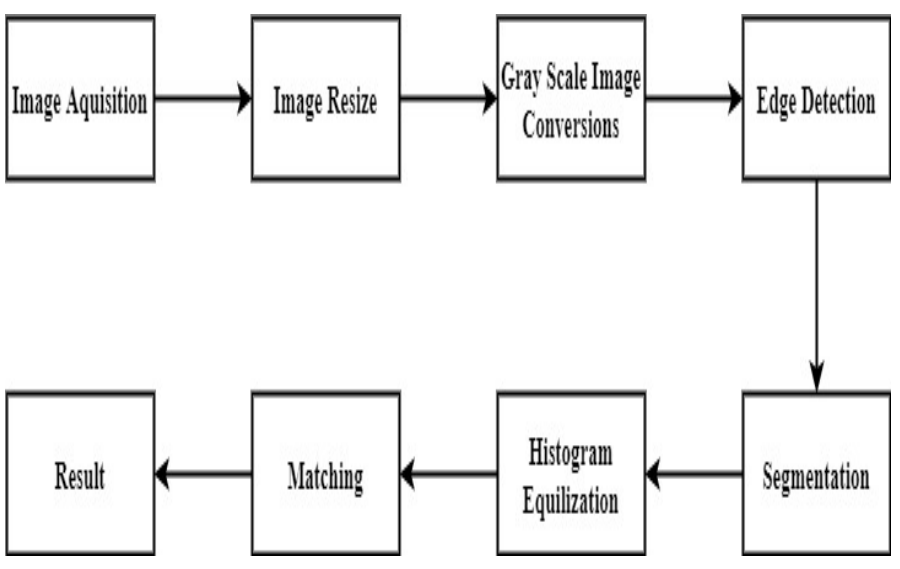

Fig 3:Block Diagram

Fig 3:shows the block diagram for the methodology used for our system

Basically it is done in 8 steps:

1)Image Acquisition 2) Image Resize 3) GrayScale Conversion

4) Edge Detection 5) Segmentation 6) Histogram Equalization

7) Matching 8) Result.

Firstly the image acquisition is done by capturing the image of

the ear from the digital camera, then the captured image goes in the internal process of resizing the image to the required size. After this the gray scale conversion of the image is done so that the image is converted into grayscale so that the information for each pixel required will be less. After gray scaling the image edge detection is done so that the boundaries of the ear shape are highlighted which will help in image processing and also after edge detection the image segmentation can be done. In image Segmentation the required areas of our image is extracted in our case it would be helix, anti helix, lobe, anti-lobule, etc.so that the further processing can be done in that region and also segmentation of the images will remove noisy edges from the image and will help to perform analysis on the image. Histogram Equalization of the image is done to process the image and is used to improve the contrast in the images to distinguish the foreground part of the image from the background part of the image and to make the image more clearer. The matching of the image is done i.e the stored image from the database is matched against the processed image and accordingly the result is displayed whether the user is identified as authorized user or not.

\section{CONCLUSION}

In our system the process of ear recognition is based on the results obtained from preprocessing of the image of the ear. By using edge detection, feature extraction and classification 
techniques to obtain the processed image of the ear. This system will help to recognize the users uniquely as ear is the perfect source of data to identify/verify the authorized user. In our daily lives there are many places which require authentication of an individual for many purposes such as ATM, attendance system, unlocking our phones and many more. So for this purpose ear as a biometric will help a lot due to unique property and as the size of ear is large it can be captured with minimal attention unlike other systems which requires numerous attempts because of the attention it needs to get scanned, also there will be minimum risk of system getting hacked and also your account or personal information will be safe.

\section{REFERENCES}

[1] Prof. Bandyopadhyay Samir Kumar,(2016), "Ear based biometric authentication system", WJERT, Vol. 2 , Issue 5.

[2] Asmaa Sabet Anwar (2015), "Human Ear Recognition using geometrical feature extraction", Procedia Computer

Science $65529-537$.

[3] Tandel Gopal Singh (2014), "Human Recognition through ear biometrics using average ear approach", IJRDET,(ISSN 2347 - 6435 (Online)).

[4] Gore Dayanand B, "Ear Recognition Technique for Biometrics using Digital Image Processing: A Review”, IOSR Journal, PP 22-26.

[5] Prof. Kalal Ashok Kumar (2017), "Biometric ear recognition system", IRJET, Volume: 04 Issue: 1.

[6] A. Kumar, C. Wu, (2011), "Automated human identification using ear imaging”, Pattern Recognition, doi:10.1016/j.patcog.2011.06.005

[7] F Liu,D Zhang . (2014), “3D fingerprint reconstruction system using feature correspondences and prior estimated finger model. Pattern Recognition" ,47(1):178-93

[8] M Yaqubi, K Faez, S Motamed (2008), "Ear Recognition Using Features Inspired by Visual Cortex and Support Vector Machine Technique". In: International Conference on Computer and Communication Engineering (ICCCE), p. 533 -537.

[9] S. A. Daramola, O. D. Oluwaninyo, (2011), "Automatic ear recognition system using back propagation neural network", International Journal of video \& image processing and network security, IJVIPNS-IJENS, Vol. 11, No.1, pp.28-32.

[10] Khursheed Farida, A.H. Mir, (2014), "AR Model Based Human identification using Ear Biometrics", IJSIP, Vol.7, No3,pg.347-360.

[11]Dhumal Yashomati,Karkud Madhuri,Shinde Pooja,Alhat Varsha,(2016), "The Human Identification System Using Geometrical Feature Extraction Of Ear ”,IJARCSSE, Vol.6, Issue 3,ISSN:2277 128X, pg.298303.

[12] M. Burge, and W. Burger (2002), "Ear biometrics in computer vision", Proc. ICPR 2000,pp. 822-826.

[13]Pug Anika \& Busch Christoph, (2012), "Ear Biometrics: A Survey of Detection, Feature Extraction and Recognition Methods"IET Biometrics, Institution of Engineering and Technology, Darmstadt, Germany, pp 1-35.

[14] M Alaraj, J Hou, and T Fukami (2010). “A Neural Network Based Human Identification Framework Using Ear Images.' In Proceedings of TENCON 2010 Conference. Fukuoka; p. 1595-1600.

[15]M. A. Turk and A. P. Pentland,(1991). "Face Recognition using Eigenfaces", IEEE Conference on Computer Vision and Pattern Recognition, pp.586-591.

[16]Pun K. and Moon Y. (2004), "Recent advances in ear biometrics. In Automatic Face and Gesture Recognition",.Proceedings. Sixth IEEE International Conference on, pages 164 - 169, May 2004.

[17] Singh Durgesh, Singh Sanjay K.(2013), "A Survey on Human Ear Recognition System Based on 2D and 3D Ear Images" OPEN JOURNAL OF INFORMATION SECURITY AND APPLICATIONS ISSN (Print): 2374-6262 ISSN(Online): 2374-6289, Volume 1, Number 2, September 2013.

[18]Chen, Hui, and Bir Bhanu, (2007), "Human ear recognition in 3D." Pattern Analysis and Machine Intelligence, IEEE Transactions on 29.4 718-737. 